\title{
TEMPERATURE APPROACH OPTIMIZATION IN THE DOUBLE PIPE HEAT EXCHANGER WITH GROOVE
}

\author{
Putu Wijaya Sunu, Daud Simon Anakottapary, Wayan G. Santika \\ Department of Mechanical Engineering, Bali State Polytechnic, Badung-Bali, Indonesia. \\ E-mail: wijayasunu@pnb.ac.id
}

\begin{abstract}
Heat transfer in double pipe heat exchanger with circumference-rectangular grooves has been investigated experimentally. The volume flowrate of cold and hot water were varied to determine its influence on the approach temperature of the outlet terminals. In this experimental design, the grooves were incised in annular room that is placed on the outside surface of the inner pipe. The shell diameter is $38.1 \mathrm{~mm}$ and tube diameter $19.4 \mathrm{~mm}$ with $1 \mathrm{~m}$ length, which is made of aluminum. The flow pattern of the two fluids in the heat exchanger is a parallel flow. The working fluid is water with volume flow rate of 27.1, 23.8 and $19.8 \mathrm{l} /$ minute. The temperature of water on the inlet terminals are $50 \pm 1^{\circ} \mathrm{C}$ for hot stream and $30 \pm 1{ }^{\circ} \mathrm{C}$ for cold stream. Temperature measurements conducted on each terminal of the inlet and outlet heat exchanger. The results showed that the grooves induced the approach temperature. The change of the approach temperature from the grooves compared to that of without grooves decreased by $37.9 \%$. This phenomenon indicates an increase in heat transfer process and performance of the heat exchanger. Groove improves the heat surface area of the inner pipe, increasing the momentum transfer and in the other hand, reducing the weight of heat exchangers itself.
\end{abstract}

Keywords: Approach temperature, groove, heat exchanger, optimization

\section{INTRODUCTION}

A heat exchanger is a heat transfer device that exchange heat between two or more process fluids at different temperature. Heat exchangers have widespread applications in industrial such as industry process, petroleum transportation, power, heat recovery, RHVAC and many domestic applications. One of the simply heat exchanger is double pipe heat exchanger (DPHE). Their usual application is for small duties and they are suitable for high pressure and temperature. Another advantage is the design of DPHE serviceable easily than the other heat exchanger and can be connected in series or parallel. Heat exchangers have to fulfill some requirement such as effective temperature difference. The effective temperature driving force is a measure of the potential for heat transfer that exists at the design conditions. Effective temperature difference is defined by the log mean temperature difference (LMTD). Other simply method to measure thermal performance of heat exchanger is using temperature approach. Temperature approach referred to difference of temperature between hot and cold outlet of heat exchanger.

The need for lightweight, space-saving, and economical heat exchangers has driven the development of compact surfaces. Many engineering techniques have been developed for enhancing the rate of heat transfer through the wall surface of heat exchanger. The enhanced of heat transfer accompanied by pressure drop and drag increase. One of the flow surface applications to increase heat transfer in turbulence flow is groove. Using grooves is well known as passive flow control. The passive flow control as concerned wide consideration as it need no auxiliary power, increase surface area, induced pressure drop, simplicity and hence give more benefits (Sunu et al., 2015). Changing the surface of pipe with circumference rectangular groove can be used to control flow separation. The groove provided as vortex generator and increased the boundary layer momentum.

Over the years, numerous researchers have carried out experimental studies on flow characteristics through internally grooved pipe. Sunu (2015) investigated increased pressure drop on internal wall surface in pipe with rectangular groove. Sunu et al (2015) revealed the effect of groove numbers on pressure drop in pipe flows. Investigation about pressure drop characteristics in a periodically grooved was conducted by Adachi et al (2009). The investigation on pipe bend with rough surface produced $4 \%$ drag reduction (Nakao, 1995). Lee and Jang (2005) studied v-shaped groove over curved body. Choi and Orchard (1997) reveal about turbulence management using passive device for momentum transfer. Ding et al (2009) present a correlation to predict frictional pressure drop of R410A-oil mixture inside internal spiral grooved microfin tube. Alam and Yong Kim (2012) conducted mixing analysis in microchannel with rectangular grooves. A number of studies have been carried out to optimize the enhancement the heat transfer of rough surface geometry. Investigation of turbulent channel flow conducted by Katoh et al (2000), reveal the relationship between drag and thermal enhancement by rough surface. Aroonrat et al (2013) investigated the relationship of heat transfer and flow characteristics in horizontal internally grooved tube made by stainless steel. The result showed the thermal enhancement factor is 1.4 to 2.2 for grooved tube. Graham et al (1999) studied of pressure drop and heat transfer in copper tube with axially grooved. Lorenz et al (1995) investigated the distribution of the heat transfer coefficient in a channel with periodic transverse grooves.

In the several paper describe above, most studies focus on heat transfer, flow characteristics and the both relationship inside/outside grooved tube. The studies of heat transfer through grooved pipe in a room between tube and shell has received little attention. Consequently, the objective of this present study is to optimize the temperature approach of double pipe heat exchanger with groove placed in annulus room.

\section{METHODOLOGY}

The flow pattern of the two fluids in a general heat exchanger can vary depending on the heat exchanger 
design. One of the flow patterns is a parallel flow, which is the hot fluid and cold fluid move in the same direction. This investigation used parallel flow in the flow direction. The hot side inlet temperature is in contact with the cold side inlet temperature, the two fluid progresses through the heat exchanger and at the other terminal point the cold side outlet temperature is in contact with the hot side outlet temperature. In a parallel flow heat exchanger, the approach temperatures are the difference between the outlet temperature of one stream and the outlet temperature of the other stream

The experiment was performed using the experimental apparatus shown schematically in Fig.1. The experiment installation consisted two centrifugal pump which was stabilized by electric stabilizer to circulate hot and cold water through a loop. The control valve used to vary flow rate through the test section and the water tank to hold water from the system.

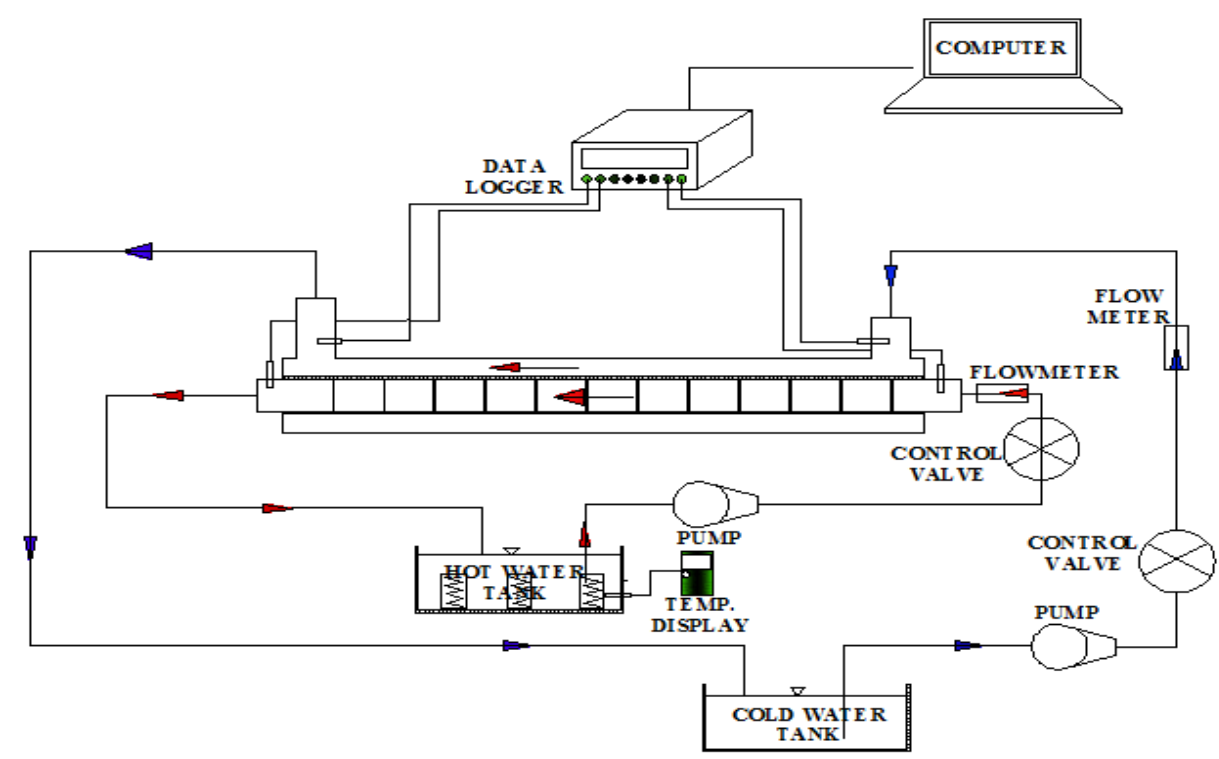

Figure-1. Experiment set-up

The hot water temperature was $50 \pm 1{ }^{\circ} \mathrm{C}$ and the cold water was $30 \pm 1{ }^{\circ} \mathrm{C}$. The volume flow rate was varied by control valve set some distance from test section as 27.1, 23.8 and $19.81 /$ minute respectively. The tube test outer diameter is
$19.4 \mathrm{~mm}$ which has circumference rectangular groove $40 \mathrm{x}$ $0.4 \mathrm{~mm}$. While, the shell test section has $38.1 \mathrm{~mm}$ outer diameter. All of the tube made of aluminum pipes.

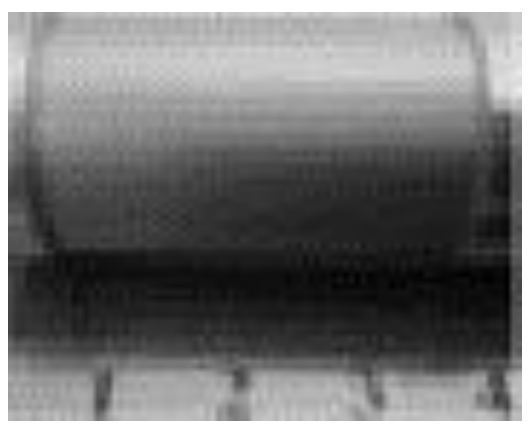

Figure-2. Groove geometry

Temperatures were measured by thermocouples placed in the inlet and outlet terminal of hot and cold water from the test section. A data logger digitalized signal from thermocouples before being recorded in a computer memory. The approach temperature (AT) was determined using the data point from hot and cold terminals of $750 \mathrm{~s}$.
The percentage of approach temperature (\% AT) from the grooves was compared to that of a smooth pipe (without grooves) and estimated by using the equation bellow:

$\% A T=\left[\frac{(A T)_{\text {smooth }}-(A T)_{\text {groove }}}{(A T)_{\text {smooth }}}\right] x 100 \%$ 


\section{RESULTS AND DISCUSSIONS}

Preliminary experiments were done on the double pipe heat exchanger without groove (smooth pipe) to establish the time series temperature and approach temperature.
This data was then used as a comparison to the data from the double pipe heat exchanger with groove. The approach temperature data in DPHE are shown in fig. 3 and 4.

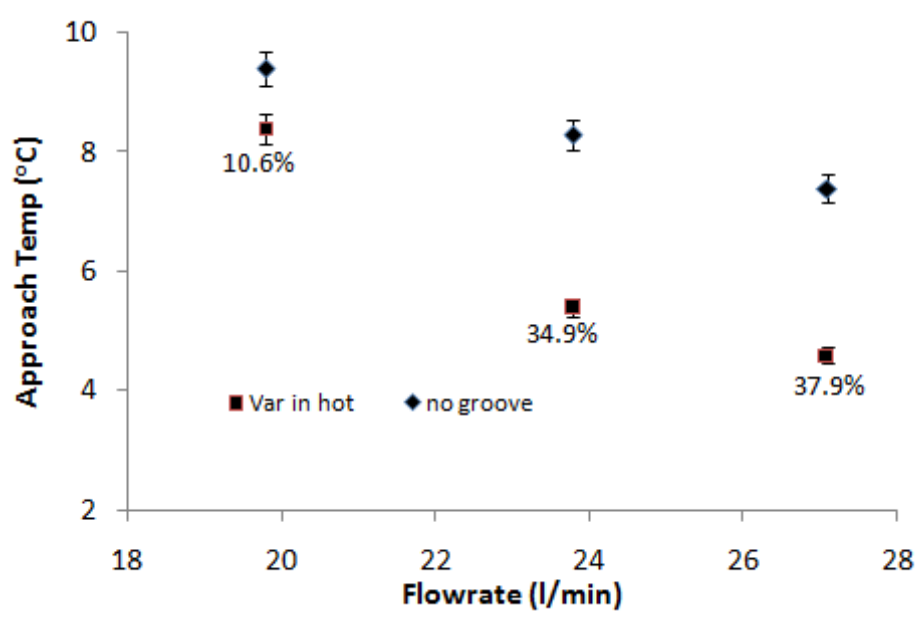

Figure-3. Average approach temperature for hot water flowrate variation.

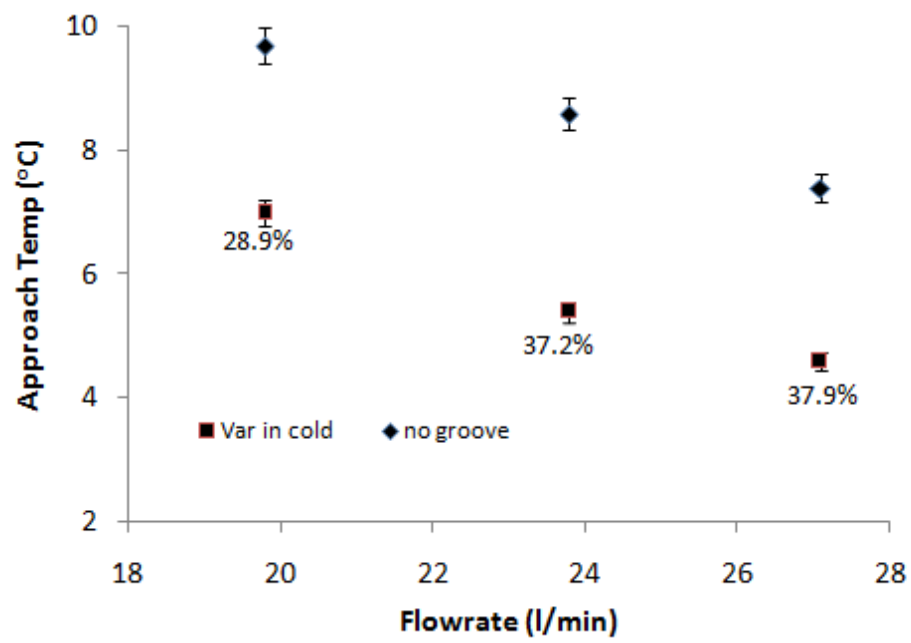

Figure-4. Average approach temperature for cold water flowrate variation.

Fig. 3 shows the average value of approach temperature at various hot water flowrate with constant cold-water flowrate. While figure 4 shows the average value of approach temperature at various cold-water flowrate with constant hot water flowrate. The rectangular pointed denotes the average approach temperature in smooth pipe DPHE. The percentage values on the plot show percentage approach temperature estimated using equation 1. For variation in hot water supply 19.8, 23.8, $27.1 \mathrm{l} / \mathrm{min}$, the approach temperature data of grooved DPHE indicates reduction of about $10.6 \%, 34.9 \%$, and $37.9 \%$ respectively. For variation in cold water supply $19.8,23.8,27.1 \mathrm{l} / \mathrm{min}$, the approach temperature data of grooved DPHE indicates reduction of about $28.9 \%$, $37.2 \%$, and $37.9 \%$ respectively. This phenomenon indicates the increases of heat transfer process between two fluids.
It was observed that approach temperature decreased with increasing flow rate in all case. The approach temperature obtained from the circumferencerectangular grooved was lower than that those from the smooth pipe. This occurred because the grooved pipe provided the enhancement of heat transfer by increasing recirculation region of the flow. Groove produced swirl effect on the fluid near to the pipe surface in the annulus room which lacerating the boundary layer. The fluid thermal boundary layer became thinner and the increasing fluid mixing process increased the rate of heat transfer which represented by the rate of approach temperature. 

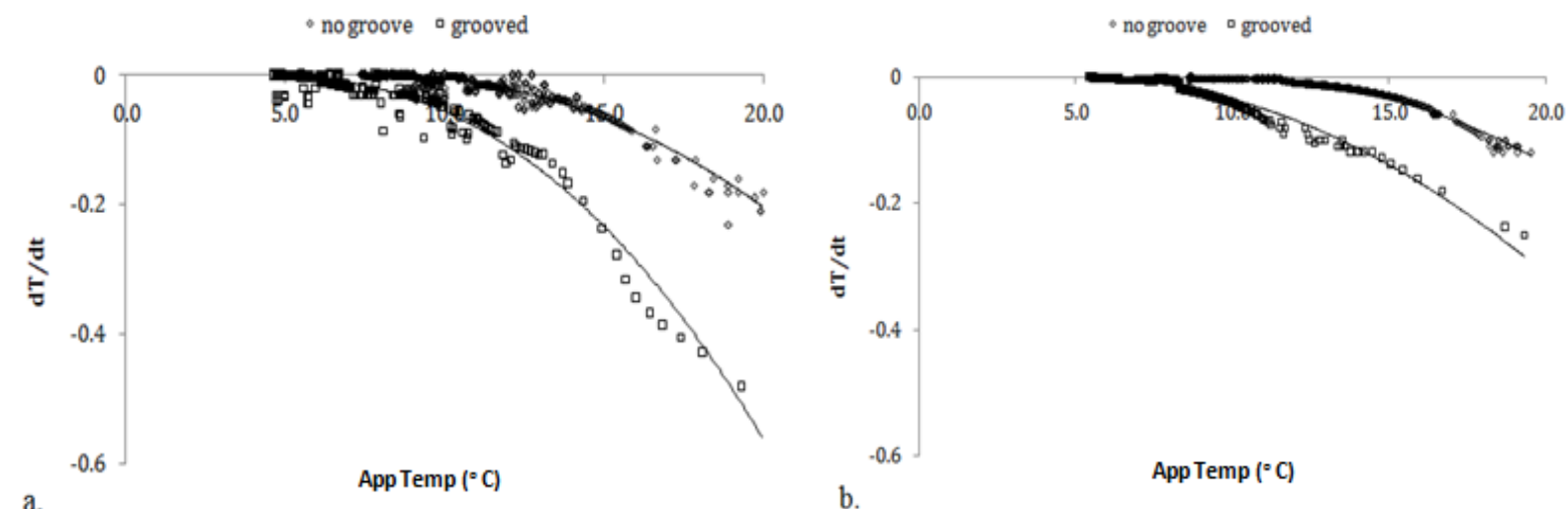

a.
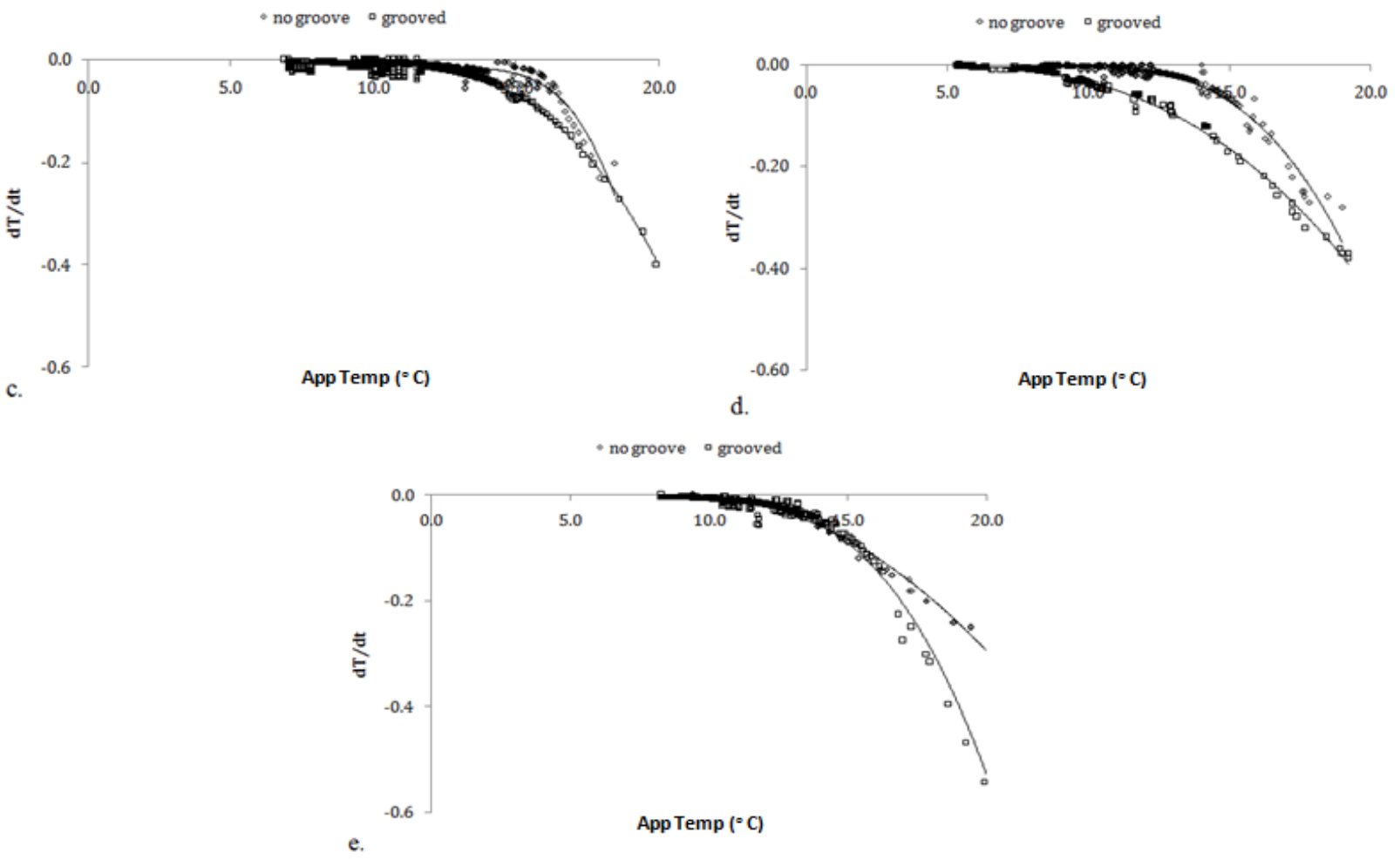

Figure-5. The rate of approach temperature (a) $\mathrm{Q}_{\text {hot }}=\mathrm{Q}_{\text {cold }}=27.1 \mathrm{1} / \mathrm{min}$; (b) $\mathrm{Q}_{\text {hot }}=27.1 \mathrm{l} / \mathrm{min}, \mathrm{Q}_{\text {cold }}=23.8 \mathrm{l} / \mathrm{min}$; (c) $\mathrm{Q}_{\text {hot }}=27.1 \mathrm{l} / \mathrm{min}, \mathrm{Q}_{\text {cold }}=19.8 \mathrm{l} / \mathrm{min}$; (d) $Q_{\text {hot }}=23.81 / \mathrm{min}, Q_{\text {cold }}=27.11 / \mathrm{min}$; (e) $Q_{\text {hot }}=19.81 / \mathrm{min}, Q_{\text {cold }}=27.11 / \mathrm{min}$

The rate of approach temperature as a function of approach temperature is presented in figure 5. Marked with circle indicates the condition of smooth pipe (without groove) and marked with rectangular point represent with grooved pipe. Negative sign on ordinate axis, indicating the occurrence of a decrease in approach temperature and indicates greater of heat transfer process. For $\mathrm{dT} / \mathrm{dt}$ grooved pipe, the rate of approach temperature for each approach temperature is more negative then that the smooth pipe. The greater the flow rate, the more negative the value $\mathrm{dT} / \mathrm{dt}$ will be. Increased flowrate is accompanied by an increase in Re. Greater Re will increase the randomness of fluid motion, resulting in higher momentum convection and energy transfer through interchange of fluid particles.

\section{CONCLUSIONS}

Heat transfer in double pipe heat exchanger with circumference-rectangular grooves was investigated using experimental method. The results showed that the grooves induced the approach temperature. Groove improves the heat surface area of the inner pipe (tube side), increasing the momentum transfer, produced swirl effect on the fluid near to the pipe surface in annulus room. The change of the approach temperature from the grooves compared to that of 
without grooves decreased by $37.9 \%$, which indicates an increase in heat transfer process and performance of the heat exchanger.

\section{REFERENCES}

Adachi Takahiro, Yamato Tashiro, Hirofumi Arima, Yasuyuki Ikegami. 2009. Pressure drop characteristics of flow in a symmetric channel with periodically expanded grooves. Chemical Engineering Science 64: 593-597

Alam Afroz, Kwang-Yong Kim. 2012. Analysis of mixing in a curved microchannel with rectangular grooves. Chemical Engineering Journal 181-182: $708-716$.

Aroonrat, K., Jumpholkul, C., Leelaprachakul, R., Dalkilic, A.S., Mahian, O., Wongwises, S. 2013. Heat transfer and single-phase flow in internally grooved tube. International Communication in Heat and Mass Transfer 42: 62-68.

Choi, K.S and Orchard D.M. 1997. Turbulence Management Using Riblets for Heat and Momentum Transfer. Experimental Thermal and Fluid Science 15: 109-124.

Ding G., Haitao Hu, Huang X., Bin Deng, Yifeng Gao. 2009. Experimental investigation and correlation of two-phase frictional pressure drop of R410A-oil mixture flow boiling in a $5 \mathrm{~mm}$ microfin tube. International Journal of Refrigeration 32: 150161.

Graham D., J.C. Chato, T.A. Newell. 1999. Heat transfer and pressure drop during condensation of refrigerant $134 \mathrm{a}$ in an axially grooved tube, International Journal of Heat and Mass Transfer 42: 1935-1944.

Katoh K., Choi, K.S.,Azuma, T. 2000. Heat-transfer enhancement and pressure loss by surface roughness in turbulent channel flows. International Journal of Heat and Mass Transfer, 43: 4009-4017.

Lee, S.J. and Jang, Y.G. 2005.Control of flow around a naca 0012 airfoil with a micro-riblet film. Journal of Fluid and Structure 20: 659-672.

Lorenz S., Mukomilow D., Leiner W. 1995. Distribution of the heat transfer coefficient in a channel with periodic transverse grooves. Experimental Thermal and Fluid Science 11: 234-242
Nakao, S.I. 1995. Effects of riblet bends on pipe flows. Applied Scientific Research 54: 237-247.

Sunu P.W. 2015. The characteristics of increased pressure drop in pipes with grooved. Adv. Studies Theor. Phys. 9(2): 57-61. http://dx.doi.org/10.12988/astp.2015.412152

Sunu P.W., ING Wardana, A.A.Sonief, Nurkholis Hamidi. 2015. The effect of wall groove numbers on pressure drop in pipe flows. Int. J. Fluid Mech. Resch., 42(2): 119 - 130 